INTERNATIONAL JOURNAL OF SCIENTIFIC RESEARCH

\title{
JUVENILE GIANT FIBROADENOMA: A CASE REPORT
}

\section{General Surgery \\ Dr. R. \\ Jayaraghavan}

General Surgery Resident in BJGMC pune

\section{ABSTRACT}

Giant juvenile fibroadenomas are rare variants of fibroadenoma, usually occurring in girls between the ages of 10 and 18 years. They are characterized by massive and rapid enlargement of a rubbery, mobile, and non-tender mass. The etiology is not well understood and believed to be an increased sensitivity to normal estrogen level. We report a case of giant juvenile fibroadenoma in a 17 years old girl that was managed by surgical excision with conservation of normal breast tissue, nipple, and areola.

\section{KEYWORDS}

\section{INTRODUCTION}

Breast development is an early physical change during puberty. Breast masses in children and adolescents are commonly benign in etiology with fibroadenoma being the most common pathological diagnosis Fibroadenoma is termed juvenile if it occurs in children and adolescents between the ages of 10-18 years. A rare variant is giant fibroadenoma characterized by a rapidly growing tumor with a mass greater than $5 \mathrm{~cm}$ and/or weighing more than $500 \mathrm{~g}$. Juvenile fibroadenoma forms only $4 \%$ of the total fibroadenomas, and giant juvenile fibroadenoma constitutes only $0.5 \%$ of all fibroadenomas. The etiology of the disease is still not well understood, but hormonal factors have been proposed. Here we present a case of giant juvenile fibroadenomas in a 17 yr old girl.

\section{Case Report}

A 17-year-old girl without any previous medical history, presented with a 3 months history of painless, palpable, and progressively enlarging mass in the left breast. There was no history of trauma, fever, anorexia or weight loss. Breast examination revealed a 8 x $7 \mathrm{~cm}$ wellcircumscribed and mobile mass in the left breast, while the other breast was normal. The mass was firm, round, and not fixed to the underlying structure. The skin was tense with prominent superficial veins. No palpable axillary lymphadenopathy was detected and there were no positive findings in the laboratory examination. Family history was also negative. On physical examination, her vital parameters were normal. She reached menarche at 13 yrs.Blood count, biochemical tests were in normal limits.

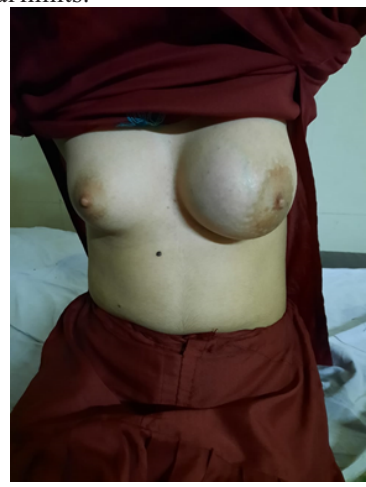

Ultrasonography reveals $4.2 * 9.8 * 7.4 \mathrm{~cm}$ well defined circumscribed parallel heterogenous predominantly hypoechoic solid lesion noted in upper inner and upper outer quadrant of left breast extending from $10^{\prime}$ oclock to 2 'oclock and classified as BIRADS Stg iii. Fine needle aspiration cytology reveals benign breast disease( fibroadenoma). Total excision of mass done with preservation of normal breast tissue, skin and nipple areola complex. Macroscopically, greyish white well encapsulated mass measuring $8 * 7 * 5 \mathrm{~cm}$.

On cut section greyish white in colour having slit like spaces. Microscopically well circumscribed tumour showing proliferating gland and stroma arranged in both pericanalicular and intracanalicular pattern suggestive of firoadenoma. Post operative recovery was uneventful.
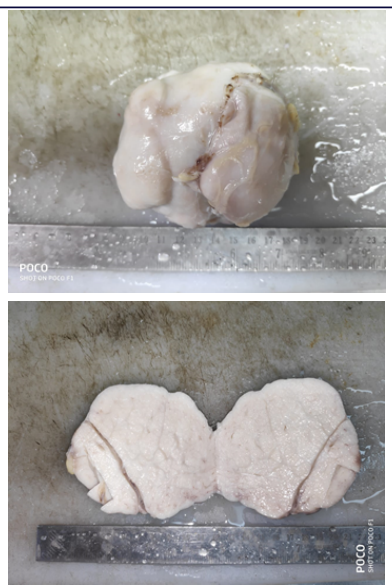

\section{DISCUSSION}

Fibroadenomas are benign solid tumor associated with aberration of normal lobular development. Although simple fibroadenoma is a disorder of breast development, giant fibroadenoma is a disease. Important differential diagnosis are: phyllodes tumor and juvenile gigantomastia. Phyllodes tumor is an uncommon fibroepithelial tumor of the breast which is more likely to occur in women over 35 years.Phyllodes tumors may be separated from GJF with their increased mitotic activity in histological examination. They can be rarely malignant and the surgical choice should be excision with preservation of breast. Juvenile gigantomastia is characterized by its positive estrogen receptor status and its hypersensitivity to estrogen. Besides phyllodes tumor, juvenile breast hypertrophy, giant lipoma, hamartoma of breast, huge cysts should be kept in mind in differential diagnosis.

Giant fibroadenomas may be classified as juvenile and adult in terms of age and simple and complex of histological appearance. About $90 \%$ of the tumors are low grade or benign and so, juvenile fibroadenomas should be treated by lumpectomy with conservation of normal breast tissue; small lesions can be treated by simple enucleation from an circum- areolar incision.

\section{CONCLUSION}

Pre-operative diagnosis of juvenile giant fibroadenoma is difficult High index of suspicion is required for the diagnosis. Surgical excision reains the cornerstone of the treatment. The challenge to the physician is to differentiate it from phyllodes which may require aggressive treatment.

\section{REFERENCES}

1. Yagnik VD. Juvenile giant fibroadenoma. Clin Pract. 2011 Jul 1;1(3):e49. doi 10.4081/cp. 2011.e49. PMID: 24765310; PMCID: PMC3981390.

2. Sosin M, Feldman E. Giant juvenile fibroadenoma: a case and review of novel modalities in treatment. Breast Dis. 2012;34(1):35-8. doi: 10.3233/BD-130342. PMID: 23443071

3. Çelik MF, Dural AC, Ünsal MG, Akarsu C, Alim ER, Kapan S, Kalaycı MU, Alış H Giant juvenile fibroadenoma. Ulus Cerrahi Derg. 2014 Dec 25;31(2):96-8. doi: 10.5152/UCD.2014.2574. PMID: 26170749; PMCID: PMC4485822 